\title{
The Pentose Phosphate Pathway in Cancer: Regulation and Therapeutic Opportunities
}

\author{
Noorhan Ghanem ${ }^{a} \quad$ Chirine El-Baba ${ }^{a} \quad$ Khaled Araji $^{a}$ Riyad El-Khoury ${ }^{b}$ \\ Julnar Usta ${ }^{a}$ Nadine Darwiche ${ }^{a}$ \\ aDepartment of Biochemistry and Molecular Genetics, American University of Beirut, Beirut, Lebanon; \\ ${ }^{b}$ Department of Pathology and Laboratory Medicine, American University of Beirut, Beirut, Lebanon
}

\section{Keywords}

Cancer - Metabolism · Pentose phosphate pathway

\begin{abstract}
Background:Tumorigenesis is associated with deregulation of nutritional requirements, intermediary metabolites production, and microenvironment interactions. Unlike their normal cell counterparts, tumor cells rely on aerobic glycolysis, through the Warburg effect. Summary: The pentose phosphate pathway (PPP) is a major glucose metabolic shunt that is upregulated in cancer cells. The PPP comprises an oxidative and a nonoxidative phase and is essential for nucleotide synthesis of rapidly dividing cells. The PPP also generates nicotinamide adenine dinucleotide phosphate, which is required for reductive metabolism and to counteract oxidative stress in tumor cells. This article reviews the regulation of the PPP and discusses inhibitors that target its main pathways. Key Message: Exploiting the metabolic vulnerability of the PPP offers potential novel therapeutic opportunities and improves patients' response to cancer therapy.
\end{abstract}

(c) 2021 S. Karger AG, Basel

Noorhan Ghanem and Chirine El-Baba contributed equally.

\section{Introduction}

Cancer cells alter their metabolism in order to sustain their rapid proliferation and survival [1]. The hallmarks of cancer highlight the complexity of the multistep process of tumorigenesis $[2,3]$ that includes both genetic and epigenetic alterations [4]. Cancer hallmarks include enhanced proliferative signaling, evading programmed cell death, avoidance of growth suppressors, unlimited replicative potential, induction of angiogenesis, tissue invasion and metastasis, escaping immune destruction, and reprograming cellular metabolism and bioenergetics [2]. Targeting cancer metabolism has gained interest over the past decade [5]. Indeed, the higher rates of energy demand in cancer cells involve the alteration of protein, lipid, carbohydrate, and nucleic acid metabolism. In addition, the abnormal tumor microenvironment induces stressors such as hypoxia, low $\mathrm{pH}$, and nutrient deprivation that elicit cancer metabolism alteration [6]. The uncontrolled proliferation of cancer cells requires an increased import of building blocks to support tumor progression. Glucose and glutamine, the principal growth-supportive substrates, provide intermediates that are diverted into branching pathways that fuel adenosine triphosphate (ATP), nicotinamide adenine dinucleotide

Correspondence to:

Nadine Darwiche, nd03@aub.edu.lb 
(NADH), and nicotinamide adenine dinucleotide phosphate $(\mathrm{NADPH})$ production $[7,8]$. These molecules are essential for nucleotide and nonessential amino acids assembly as well as for fatty acid and organelle biosynthesis [9]. Cancer cells rewire their metabolism and rely on high rates of aerobic glycolysis to meet their extra growth and maintenance requirements [10].

The pentose phosphate pathway (PPP) is the first among a series of pathways that branch from glycolysis. The PPP is a major glucose catabolic and reducing anabolic pathway essential for neoplastic transformation due to the production of NADPH and nucleic acid precursors [11]. Besides upregulating the PPP, tumor cells aberrantly activate oncogenes and/or repress tumor suppressor genes that lock cancer cells in a constitutively scavenging state. Notably, the Ras and the phosphoinositide 3-kinase/protein kinase B/ mammalian target of rapamycin signaling pathways induce the overexpression of the plasma membrane glucose transporter 1 (GLUT1) and hexokinase, which is the first enzyme of glycolysis [12]. This marked increase in glucose consumption by tumors compared to healthy counterpart tissue is known as the Warburg effect, where the majority of glucose is transformed into lactate [13]. Otto Warburg reported that in most cancer cells, even in the presence of oxygen, tumor cells favor and reprogram their glucose metabolism to rely largely on glycolysis for energy production, resulting in a state of "aerobic glycolysis" [14]. However, under aerobic environment, normal cells process glucose via glycolysis into pyruvate that is further metabolized in the mitochondria into carbon dioxide, ATP, and water. Although there are several metabolic pathways that are deregulated in cancer [15], this review focuses on the involvement of the PPP in cancer cell proliferation and survival, and its inhibition in cancer therapeutics.

\section{The Pentose Phosphate Pathway: Oxidative and Nonoxidative Phases}

The PPP, also known as the hexose monophosphate shunt or the phosphogluconate pathway, is a pivotal pathway that is induced in neoplasms [16]. The PPP contributes to ribonucleotide synthesis and maintains cytosolic reduction-oxidation (redox) balance through NADPH production. Depending on cellular metabolic needs, the PPP may utilize intermediates from the tricarboxylic acid, lipid, and amino acid metabolic pathways. The first PPP enzyme, glucose-6-phosphate dehydrogenase (G6PD), gained significant interest for its involvement in hemolytic anemia, a condition induced by oxidizing agents, such as fava beans. Moreover, red blood cells are rendered vulnerable to oxidative stress in patients with G6PD deficiency as the PPP is their unique source of NADPH and plays a major antioxidant role by scavenging reactive oxygen species (ROS) [17]. With over 400 million people suffering from genetic defects in $\mathrm{G} 6 \mathrm{PD}$, it is interesting to study its correlation with a lower risk of cancer as G6PD may act as an oncogene in cancer cells [18].

The PPP is composed of 2 phases: the irreversible oxidative and the reversible nonoxidative (shown in Fig. 1). The oxidative phase involves 3 enzymatic reactions that generate 2 NADPH molecules and ribulose-5-phosphate (Ru5P). The first substrate, glucose-6-phosphate, is oxidized to 6-phosphogluconate (6 PG) by G6PD, yielding the first NADPH molecule. Subsequently, 6 PG undergoes oxidative decarboxylation by 6-phosphogluconate dehydrogenase (6PGD) producing Ru5P and the second $\mathrm{NADPH}$ molecule. G6PD catalyzes the rate determining step in the oxidative phase, highlighting its firmly regulated activity. In rapidly proliferating cells, a high $\mathrm{NADP}^{+} /$ NADPH ratio positively modulates the activity of G6PD to support NADPH production. NADPH is an essential anabolic reducing agent that supports reductive biosynthesis of fatty acids and nucleotides. Furthermore, NADPH maintains cell survival under oxidative stress conditions that are generated from deregulated mitochondria or metabolically active cells [19]. Glutathione (GSH) is an essential antioxidant tripeptide that scavenges ROS, and NADPH is a critical cofactor involved in continuously replenishing the GSH pool [20]. Enhanced G6PD activity was reported in papillary thyroid carcinoma [21], colorectal [22], renal [23], and prostate cancer [24]. In fact, G6PD activity is upregulated by several oncogenes, such as platelet-derived growth factor, epidermal growth factor, phosphoinositide 3-kinase, and Ras $[25,26]$. On the other hand, p53 was reported to be a negative modulator of G6PD by binding directly to it and preventing monomer dimerization and enzyme activation [27]. 6PGD has also been frequently studied with G6PD for its pivotal role in tumorigenesis. In fact, genetic silencing of 6PGD led to the accumulation of cellular $\mathrm{p} 53$ proteins, resulted in senescence of lung cancer cells, and slowed tumor growth in mouse xenograft models [28].

The reversible nonoxidative phase starts with Ru5P that is transformed into ribose-5-phosphate (R5P) by ribulose-5-phosphate isomerase. R5P is an essential component of purine and pyrimidine nucleotides biosynthesis. Ru5P may also be converted into xylulose-5-phosphate by ribulose-5-phosphate-3-epimerase, which was reported to enhance glycolytic flux [17]. The nonoxida- 
Fig. 1. Schematic representation of the oxidative and nonoxidative phases of the PPP as well as various inhibitors of specific enzymes that may be targeted in cancer treatments as covered in this review. 6-AN, 6-aminonicotinamide; DHEA, dehydroepiandrosterone; miRNA, microRNA; 6PGD, 6-phosphogluconate dehydrogenase; G6PD, glucose-6-phosphate dehydrogenase; PPP, pentose phosphate pathway; TKT, transketolase; TALDO, transaldolase; G6P, glucose-6-phosphate; Ru5P, ribulose5-phosphate; 6 PG, 6-phosphogluconate; RPI, ribulose-5-phosphate isomerase; RPE, ribulose-5-phosphate-3-epimerase; F6P, fructose-6-phosphate; G3P, glyceraldehyde-3-phosphate; NADPH, nicotinamide adenine dinucleotide phosphate.

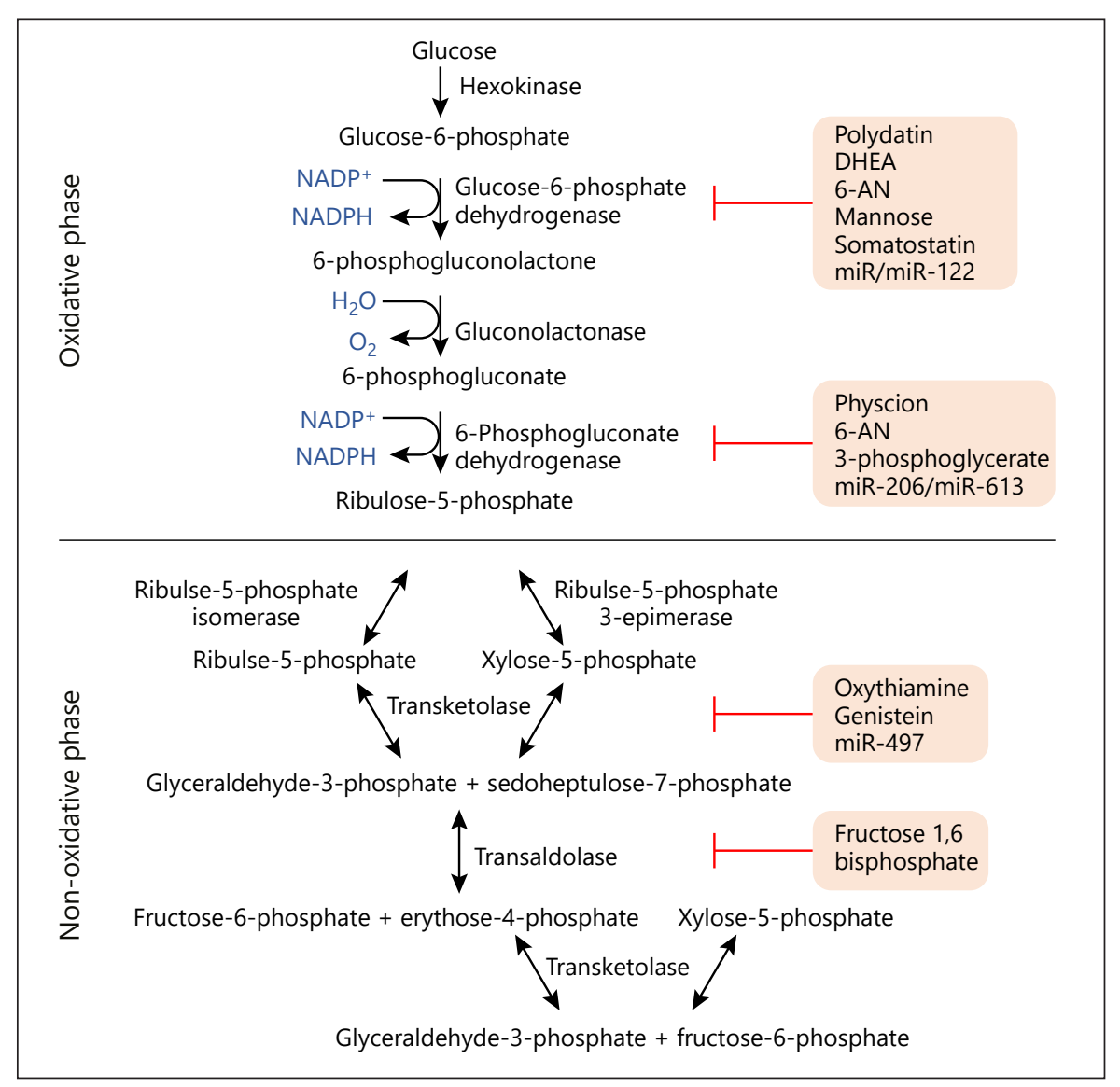

tive PPP phase is controlled by transketolase (TKT) and transaldolase (TALDO). The reversible nature of these enzymes allows glycolytic metabolites, such as fructose6-phosphate (F6P) and glyceraldehyde-3-phosphate (G3P), to be recruited into this pathway and vice versa. It has been reported that the nonoxidative PPP enzymes are upregulated in cancer [11]. In a pancreatic cancer mouse model, K-Ras stimulation was shown to activate the nonoxidative phase of the PPP [29]. TKT overexpression in lung, prostate, and breast cancer cells has been correlated with tumor invasiveness and poor cancer prognosis [17, 30]. TALDO has been shown to be elevated in several tumors, such as squamous cell carcinomas [31] and hepatocellular carcinoma [32].

\section{Regulation of the Pentose Phosphate Pathway}

The PPP enzymes can be allosterically regulated by their own metabolic products to modulate enzymatic activity and facilitate cellular growth. Depending on cellular needs and environmental cues, the PPP can operate in different modes. In rapidly dividing cells, TKT and TALDO reversely channel G3P and F6P from glycolysis to the nonoxidative PPP to generate RNA and DNA precursors from R5P. During oxidative stress, hexose phosphate isomerase converts F6P into G6P through glycolysis and shuttles it back to the PPP to maintain NADPH homeostasis. Similarly, under high ATP demand, the PPP products can be reshuffled into the glycolytic pathway [33]. When glucose availability is scarce, pathways other than the PPP maintain NADPH thresholds which are essential to mitigate oxidative stress and prevent cell death. One of these pathways is mediated by the activation of AMP-activated protein kinase that halts the consumption of NADPH by fatty acid synthesis. Simultaneously, the malic enzyme and the cytosolic isocitrate dehydrogenase induce fatty acid oxidation which generates more NADPH molecules [17]. In colorectal cancer, an increase in $\mathrm{NADP}^{+}$content due to the loss of G6PD activity is compensated by an upregulation in malic and isocitrate dehydrogenase enzymes flux to maintain a high NADPH/ 
$\mathrm{NADP}^{+}$ratio [34]. In many cancers, several factors are involved in positively regulating the flux into the PPP, namely, the proapoptotic Phorbol-12-myristate-13-acetate-induced protein 1, also known as NOXA. Interestingly, NOXA had a dual function. In the presence of glucose, NOXA facilitates the usage of the PPP to promote survival, while under scarce glucose conditions, NOXA is dephosphorylated which functions as a proapoptotic protein [35].

\section{PPP Impact on Cancer Metabolism}

Cancer cells can overcome the strict metabolic surveillance of the PPP by acquiring genetic mutations and epigenetic modifications that modulate its activity [36]. For instance, the tumor suppressor p53 stimulates the flux through glycolysis and upregulates the expression of the apoptosis regulator (TP53-inducible glycolysis and apoptosis regulator). TP53-inducible glycolysis and apoptosis regulator functions as a fructose-2, 6-bisphosphatase, thus inhibiting phosphofructokinase 1 activity, thereby redirecting glycolytic intermediates toward the PPP [37]. p53 is also known to suppress the transcription of glucose transporters GLUT1 and GLUT4. On the other hand, p21-activated kinase 4 promotes glucose uptake, NADPH production, and lipid biosynthesis. p21-activated kinase 4 is positively correlated with colorectal cancer in tissue samples, where it was shown to bind to G6PD and enhance its activity [38]. Similarly, mammalian target of rapamycin complex 1 is frequently activated in cancer cells. Its activation is often associated with a substantial upregulation in the oxidative phase of the PPP, involving particularly G6PD, by increasing the activity of the transcription factor sterol regulatory element-binding protein [39].

The activity of the PPP oxidative phase enzymes is upregulated in colorectal cancer [40] (unpublished data). 6PGD expression level and enzymatic activity are elevated in malignant hepatocellular carcinoma cells compared to normal hepatocytes [41]. Furthermore, the amount of G6PD and TKT enzymes is elevated in colorectal cancer and hepatocellular carcinoma patient tissues compared to normal counterparts $[40,42]$. Thus, exploiting the reprogrammed metabolism in tumor cells may offer novel therapeutic targets against cancer $[9,43]$. We will next cover the use of PPP inhibitors as a therapeutic window in cancer cells.

\section{PPP Inhibitors}

The PPP inhibitors can be divided into the oxidative phase or the nonoxidative phase inhibitors (Table 1).

\section{Oxidative Phase Inhibitors}

Increased flux through the PPP is observed in neoplasia and tumor invasion, and its inhibition remains a plausible attractive therapeutic approach against many cancers. Unfortunately, efficient anti-PPP agents are not available in the clinic and further investigations are still needed [44]. Listed below are a set of synthetic and natural oxidative phase inhibitors of the PPP, namely, inhibitors of G6PD and 6PGD that could be used to treat cancer and restore sensitivity to chemotherapy (Table 1). In fact, enhanced-PPP activity has been shown to be implicated in cisplatin resistance in solid tumors [45].

\section{G6PD Inhibitors}

G6PD has gained significant attention among the scientific community for its broad implications in health and disease [46]. Indeed, G6PD overexpression and elevated activity have been associated with many types of cancers. Here, we emphasize the main G6PD inhibitors in preclinical and clinical development as well as in the clinic for use as anticancer agents.

\section{Polydatin}

Polydatin $\left(3,4^{\prime}, 5\right.$-trihydroxystilbene-3- $\beta$-d-glucoside; trans-resveratrol 3- $\beta$-mono-D-glucoside; piceid) is a glucoside of resveratrol extract from the Chinese herb $\mathrm{Po}$ lygonum cuspidatum [47]. Polydatin is abundantly present in plants such as peanuts, grapes, and cocoa-containing foods. Polydatin has many important biomedical properties, such as cardioprotective activity [48], anti-inflammatory [49], immunomodulatory [50], and antioxidant effects [51]. Additionally, it has apoptotic characteristics in neoplastic cells by directly inhibiting the activity of G6PD and impairing NADPH production. In head and neck squamous cell carcinoma cells, polydatin was shown to increase ROS production, endoplasmic reticulum stress, and apoptosis while inducing cell cycle arrest and inhibiting invasion [44]. In an orthotopic metastatic tongue cancer model, polydatin led to tumor regression and repressed lymph node metastasis [44]. Moreover, polydatin revealed a synergistic effect with lapatinib, a tyrosine kinase inhibitor, in breast cancer cells, by inducing autophagy [52]. In colorectal cancer, polydatin was shown to induce cell-specific differentiation and apoptosis [53]. Currently, polydatin is in phase II clinical trials and pre- 
Table 1. Comparison between different oxidative and nonoxidative PPP inhibitors

\begin{tabular}{|c|c|c|c|c|}
\hline \multicolumn{5}{|c|}{ Oxidative PPP inhibitors } \\
\hline & DHEA & Steroid hormone & Noncompetitive inhibitor & In the clinic [137] \\
\hline & $6-\mathrm{AN}$ & Monocarboxylic acid amide & Competitive inhibitor & In the clinic [138] \\
\hline & Somatostatin & Peptide hormone & Unknown & In the clinic [139] \\
\hline & miR-1 and miR-122 & $\begin{array}{l}\text { microRNA; a type of noncoding } \\
\text { RNA }\end{array}$ & $\begin{array}{l}\text { Post-transcriptional } \\
\text { repression of 3'-UTR g6pd } \\
\text { mRNA }\end{array}$ & $\begin{array}{l}\text { miR-1: preclinical development [140-142] } \\
\text { miR-122: Phase II clinical trials [143] }\end{array}$ \\
\hline & 3-PG & $\begin{array}{l}\text { Monophosphoglyceric acid; } \\
\text { intermediate of glycolysis }\end{array}$ & Competitive inhibitor & Preclinical development [95] \\
\hline & $\begin{array}{l}\text { miR-206 and miR- } \\
613\end{array}$ & miRNA & $\begin{array}{l}\text { Post-transcriptional } \\
\text { repression of 3'-UTR } 6 P G D \\
\text { mRNA }\end{array}$ & Preclinical development $[96,145]$ \\
\hline \multicolumn{5}{|c|}{ Nonoxidative PPP inhibitors } \\
\hline TKT & Oxythiamine & Thiamine analog & $\begin{array}{l}\text { Noncompetitive irreversible } \\
\text { inhibitor }\end{array}$ & Preclinical development [146] \\
\hline
\end{tabular}

6-AN, 6-aminonicotinamide; 6PGD, 6-phosphogluconate dehydrogenase; DHEA, dehydroepiandrosterone; G6PD, glucose-6-phosphate dehydrogenase; PPP, pentose phosphate pathway; TKT, transketolase; TALDO, transaldolase; 3-PG, 3-phosphoglycerate; miR, microRNA.

liminary data show minimal side effects and high tolerance in humans, making it a natural favorable treatment [54]. However, the definitive target proteins and mode of action of polydatin remain to be elucidated.

\section{Dehydroepiandrosterone}

In 1939, the discovery of the metabolic pathway of dehydroepiandrosterone (DHEA) was awarded the Nobel Prize in Chemistry [55]. DHEA (3 $\beta$-hydroxy-5androsten-17-one) is a 19-carbon endogenous hormone synthesized de novo from cholesterol in the adrenal gland. This steroid is a precursor for estrogen and androgen [56]. Together with its sulfur ester DHEA-S, they represent the most abundant steroid in the body and peak during the second and third decades of life [55]. However, DHEA is a much more potent G6PD inhibitor than DHEA-S $[56,57]$. DHEA was originally found to have a chondroprotective role and to be effective against osteoarthritis. DHEA exerts a beneficial effect on osteoarthritic cartilage by favoring anabolic versus catabolic signaling 
pathways. For instance, DHEA upregulates the expression of the tissue inhibitor of metalloproteinases-1, while it prevents the expression and protein synthesis of metalloproteinases-1 [58]. DHEA was also found to suppress pro-inflammatory cytokines and to inhibit atherosclerosis [59]. Moreover, epidemiological analysis has shown an inversely proportional correlation between serum DHEA levels and risk of developing breast hyperplasia [60].

Besides its natural role as a hormone, research data suggest that DHEA is a strong inhibitor of mammalian G6PD [19]. The exact nature of DHEA inhibition mechanism is not yet elucidated. Some studies have shown that DHEA behave as an uncompetitive inhibitor while other studies show that DHEA may act as a noncompetitive G6PD inhibitor. It has been described that DHEA decreases cellular NADPH and increases ROS levels [61]. Moreover, many studies have indicated that DHEA was able to reduce thyroid and colorectal cancer cell proliferation [19, 62]. It should however be noted that DHEA is rapidly converted into other steroid hormones which raise concerns about its potential efficacy as an inhibitor of the oxidative phase of the PPP [56]. Clinical trials using DHEA are burdened by the elevated oral doses required. Therefore, DHEA analogs are still to be developed for a better bioavailability.

\section{6-Aminonicotinamide}

6-Aminonicotinamide (6-AN) is a monocarboxylic acid amide that is the most potent antagonist of niacin. This metabolite is converted, into its active form in vivo, resulting in analogs of $\mathrm{NAD}^{+}$and $\mathrm{NADP}^{+}$, with the latter being further completely modified into 6-AN adenine dinucleotide phosphate at much faster rate than $\mathrm{NAD}^{+}$ [63]. These analogs are incapable of participating as coenzymes in the redox reactions of human metabolism [64]. Conversion of $\mathrm{NAD}^{+}$and $\mathrm{NADP}^{+}$into their corresponding AN analogs involves substituting the nicotinamide moiety of existing $\mathrm{NAD}^{+}$and $\mathrm{NADP}^{+}$by $\mathrm{AN}$ or incorporating $\mathrm{AN}$ during the de novo synthesis of the pyridine nucleotides [65].

6-AN acts as a competitive inhibitor of the $\mathrm{NADP}^{+}$dependent G6PD and 6PGD enzymes, evidenced by 6-AN increasing the levels of ROS production while reducing NADPH biosynthesis [66]. The incubation of 6-AN with isolated hepatocytes [64] and human lung carcinoma cells [67] resulted in a marked buildup of 6 PG levels. Preclinical trials have shown that the application of 6-AN in adjuvant antineoplastic drugs potentiates the effects of radiation and chemotherapy [68], as reported in mammary carcinoma mouse models [19]. 6-AN hin- dered the growth of a panel of thyroid cancer cells [62]. Interestingly, 6-AN increased the expression of the glucose-regulated stress protein GRP78 and was correlated with enhanced response to DNA cross-linking agents in colorectal cancer [69]. Notably, the combination of cisplatin and 6-AN resulted in accumulation in platinumDNA adducts [66]. Combining 6-AN with other drugs sensitizes treatment in distinct cancers such as breast, ovarian, leukemia [70], and hepatocarcinoma [71]. In colorectal cancer, 6-AN reversed the observed increased G6PD activity. It also repressed the multidrug resistance of doxorubicin-resistant human colon cancer cells [72].

6-AN displays cytotoxic and high antiviral activity, as evidenced in several cell lines and transgenic-hepatitis B virus mouse models. 6-AN inhibited the secretion of hepatitis $B$ virus surface antigen in hepatoblastoma cells through a reduction in the peroxisome proliferators-activated receptor- $\alpha$ transcription factor activity. Previous studies have demonstrated the implication of 6-AN in treating skin diseases, namely, psoriasis. 6-AN has also shown antiparasitic responses against Leishmania and Plasmodium falciparum [73]. The use of 6-AN in the clinic is hindered by its toxicity at elevated concentrations, serious side effects, such as B-complex vitamin deficien$\mathrm{cy}$, serious neurological damage, and paralysis presumably due to its direct action on the central nervous system [74]. Further clinical research is undergoing to determine the effective doses of 6-AN to be administered to patients.

\section{Mannose}

Mannose (D-mannose) is a natural C-2 epimer of glucose imported by GLUT1 transporter [75]. Inside the cell, hexokinase phosphorylates mannose to produce mannose-6-phosphate (M6P). The latter is either isomerized by phosphomannose isomerase (PMI) into F6P, or engaged into $\mathrm{N}$-glycosylation via phosphomannosemutase 2 , or catabolized by DN-9-phosphate synthase for the synthesis of 2-keto-3-deoxy-D-glycero-D-galacto-nononic acid [76]. The fate of M6P depends on the cellular ratio of PMI to phosphomannosemutase 2. A higher ratio stimulates catabolism, while a lower ratio leads to M6P accumulation, which interferes with glucose metabolism. However, mannose slightly contributes to cellular bioenergetics as the physiological concentration of mannose in human blood is one-hundredth of that of glucose [75].

Mannose has recently captured the interest of researchers for its anticancer effect via glucose metabolism impediment $[77,78]$. Mannose showed potential anticancer effects by suppressing growth, inducing $G_{0} / G_{1}$ cell cycle arrest, promoting cisplatin-induced apoptosis, and re- 
ducing cell invasiveness in lung cancer [79]. Gonzalez et al. [78] demonstrated that mannose did not affect the intracellular levels of glucose. However, mannose was poorly metabolized inside the cell and affected several metabolic pathways. The intracellular accumulation of mannose as M6P inhibits 3 enzymes that facilitate glucose metabolism, namely, hexokinases, phosphoglucose isomerase, and G6PD [78].

Mannose showed synergistic interaction with chemotherapeutic drugs, such as cisplatin and doxorubicin, and increased their cytotoxic effect through potentiating intrinsic apoptotic pathways [78]. Therefore, mannose was suggested to be used clinically in combination with conventional chemotherapeutics agents [78, 79].

Intracellular PMI levels dictate the response to mannose. High levels of PMI are associated with mannoseinsensitive cells while depletion of PMI by RNA interference restores sensitivity to mannose [78]. Moreover, PMI overexpression eliminates the impact of mannose on glucose consumption. Notably, PMI levels varied in human tumors, which could be used as a biomarker for mannose sensitivity (unpublished data). Mannose alone or in combination with conventional cancer therapies can be used as a potential new anticancer treatment to modulate glucose metabolism in cancer cells.

Mannose receptors (MRs) are overexpressed on many cancerous cells [80]. Recently, mannose was highlighted as an anticancer drug-delivery system, which can carry chemotherapeutics to target tissues while avoiding offtarget tissues, thus minimizing side effects. Therefore, encapsulating anticancer drugs into a mannose-therapeutic system can potentially target tumors through MR-mediated endocytosis to enhance the beneficial effect while reducing toxicity [81]. Moreover, MR was found to be expressed on the surface of dendritic cells and macrophages; thus, it can be targeted to induce anticancer response and stimulate immunotherapy [80].

\section{Somatostatin}

Somatostatin is a steroid peptide hormone secreted from the hypothalamic and central nervous system regions of the human body. Although somatostatin's main function is to suppress the release of growth factor hormones, it is involved in pathophysiological and cancerrelated changes in the body [82]. Somatostatin has been documented to play antitumor effects in several animal models and human cancers cell lines and to cause glucose intolerance through G6PD suppression and TKT-catalyzed reactions in tumors [83]. Deane and Woo [83] showed that administration of somatostatin significantly

Inhibitors of the Pentose Phosphate Pathway in Cancer inhibited G6PD gene transcription and G6PD enzymatic activity in sea urchins by 70 and $52 \%$, respectively. Similarly, somatostatin was proposed to suppress the oxidative PPP phase by reducing G6PD activity, affecting ribose synthesis, and inhibiting the carbon flux into PPP [84].

\section{miR- 1 and $m i R-122$}

miR-1 has been reported to work as a tumor suppressor gene by targeting G6PD transcripts and inhibiting tumor growth and metastasis [85]. miR-1 levels are reduced in many tumors as evidenced by the increased proliferation observed in high-risk papillomavirus-associated human cervical cancer cells [86]. In cardiovascular cells, miR-1 increased oxidative stress through binding to the single 3'-UTR complementary site of G6PD and superoxide dismutase transcripts [87]. Moreover, G6PD transcripts harbor $2 \mathrm{miR}-122$ binding sites in their $3^{\prime}$-UTR regions. In HepG2 hepatocellular carcinoma cells, the combination of miR-1 and miR-122 synergistically reduced G6PD expression and its enzymatic activity [88]. These results highlight the therapeutic use of miR-1 and miR-122 by inhibiting the PPP through suppressing G6PD.

\section{PGD Inhibitors}

6PGD has been documented to be overexpressed in several cancers including colorectal [89], cervical intraepithelial [90], thyroid [91], and lung tumors [28]. 6PGD is stimulated by lysine acetylation [92]. Three potential therapeutic inhibitors of 6PGD are discussed below.

\section{Physcion}

Physcion, an active component of the medicinal plant Radix et Rhizoma Rhei, is an anthraquinone derivative, small-molecule that effectively inhibits 6PGD [91]. Purified physcion and its more soluble derivative S3 (1-hydroxy-8-methoxy-anthraquinone), inhibit purified human 6 PGD at an $\mathrm{IC}_{50}$ of approximately 39 and $18 \mu \mathrm{M}$, respectively [92]. The molecular crystal structure of 6PGD suggests that physcion binds near to the 6-PG substrate site on 6PGD [92]. Physcion interacts hydrophobically with the residues Met15, Lys 76, Lys 261, and His 452 and forms a hydrogen bond with Asn 103 in this area of the enzyme.

Physcion was shown to inhibit cisplatin-resistant ovarian C13* cancer and lung adenocarcinoma A549DDP cancer cells proliferation. In ovarian OV2008 and C13* cancer cells, the decrease in cell proliferation was associated with a reduction in 6PGD activity. Similar results 
were observed in lung adenocarcinoma A549 and A549DDP cells [92]. Physcion induced apoptosis in cervical cancer and lung carcinoma cells $[92,93]$. The cytotoxic effect of physcion on cancer cells is well tolerated in preclinical studies. This drug showed minimal effect on the proliferation of human dermal fibroblasts and on immortalized human melanocytes. Physcion significantly decreased tumor volume when administered at 20 and $40 \mathrm{mg} / \mathrm{kg} / \mathrm{day}$ in mice [94]. Moreover, the physcion pretreatment of ovarian and lung cancer cells reduced cisplatin resistance and revealed a synergistic effect of both drugs. Physcion also enhanced the activity of paclitaxel when administered in combination in xenograft models [93].

\section{3-Phosphoglycerate}

3-Phosphoglycerate (3-PG), an intermediate of glycolysis and the Calvin cycle, has been found to be a direct competitive inhibitor of 6PGD. Reports have shown that the attenuation of the glycolytic enzyme phosphoglycerate mutase 1 , which normally regulates 3 -PG levels, leads to an accumulation of 3-PG and subsequent inhibition of the oxidative PPP [95].

\section{miR-206 and miR-613}

Dysregulation of miRNA has been associated with chemotherapy resistance. For instance, miR-206 and miR-613 were found to be reduced in cisplatin-resistant cells [95]. Upon subsequent examination, the investigators found that these miRNAs are direct inhibitors of 6PGD gene expression. It is suggested that miR-206 and miR-613 have single binding domain sites on the 3'UTR of 6PGD. In combination, miR-206 and miR-613 reduced $\mathrm{NADPH} / \mathrm{NADP}^{+}$ratio, increased glycolytic lactate flux, and ATP levels [96].

\section{Nonoxidative Phase Inhibitors}

In this section, we discussed the properties of a set of synthetic and natural nonoxidative PPP inhibitors, namely, inhibitors of TKT and TALDO that could be used to treat cancer and restore sensitivity to chemotherapy (Table 1).

\section{TKT Inhibitors}

TKT is a main enzyme of the nonoxidative PPP reported to be upregulated in several cancers. TKT is essential for cancer growth due to its effect on NADPH production and on carbon intermediates transfer for nucleotides synthesis and glycolysis reentering [97]. Three main inhibitors of TKT in clinical or preclinical setting are discussed below.

\section{Oxythiamine}

Oxythiamine (4-hydroxythiamine) is an irreversible noncompetitive inhibitor of the TKT enzyme [98]. Oxythiamine is pyrophosphorylated by thiamine pyrophosphokinase giving rise to oxythiamine pyrophosphate, which has structural similarity to thiamine pyrophosphate [99]. Thiamine is an essential cofactor of the TKT enzyme; thus, oxythiamine may serve as a thiamine antimetabolite analog of this key PPP enzyme. Although its mechanism of action remains to be elucidated [100], oxythiamine seems to mainly dock onto the active site of TKT by binding to Ser49, Lys84, Glu128, His160, and Lys218 residues, with Glu128 being the preferred inhibitory site [101]. In yeast infections, oxythiamine has been used to synergize with antifungal drugs [102]. Interestingly, oxythiamine was shown to significantly suppress tumor proliferation and increase $G_{0} / G_{1}$ cell cycle arrest in Ehrlich's ascites tumor mouse models in a dose-dependent manner [103]. Oxythiamine inhibitory effects were also shown to be related with a reduction in the activity of the MAPK pathway in the pancreatic Mia PaCa-2 cells [104]. Low and high doses of oxythiamine were documented in a subcutaneous xenograft cancer model where this drug was able to impede invasion and migration of Lewis lung carcinoma and to inhibit the activity of metalloproteinases- 2 and 9 [105].

Oxythiamine has been reported to be used in combination with other drugs such as lovastatin and was shown to decrease TKT protein levels and to inhibit thyroid ARO cancer cell line proliferation [106]. Moreover, oxythiamine was shown to inhibit the carbon flux into the PPP, an effect enhanced when oxythiamine combined with somatostatin in Mia PaCa-2 cell line [84]. The combination treatment of 6-AN and oxythiamine increased DNA damage, $\mathrm{NADP}^{+} / \mathrm{NADPH}$ ratios, and ROS-induced apoptosis in thyroid cancer cell lines [62].

\section{Genistein}

Genistein is a natural soy plant-derived isoflavonoid with clinical and epidemiological significance that acts through a variety of mechanisms including antioxidant, estrogenic activities, and metabolic regulation [107-109]. It is successfully used as a tyrosine-kinase inhibitor to treat chemotherapeutic-resistant acute childhood, and chronic adulthood lymphoblastic leukemia [110]. Genistein significantly inhibited glycolysis of hepatocellular carcinoma cells by reducing the activity of hypoxia-inducible factor 1 alpha and inactivating hexokinase 2 and GLUT1 [111]. Boros et al. [112] reported that increasing dosage of genistein reduced Mia PaCa-2 cells ability to 
synthesize ribose for RNA synthesis. The mechanism of action is primarily through inhibiting the nonoxidative TKT reaction of the PPP by blocking nucleic acid synthesis. This was evident based on the mass isotope distribution analysis differential positioning of labeled carbon glucose isotopes tracing in pancreatic cancer cells upon genistein treatment [112]. In addition, genistein can prevent the proliferation of primary rhabdomyosarcoma, glioblastoma, embryonic carcinoma, and hepatocellular carcinoma cells, arrest cell cycle progression, and induce apoptosis [113]. It was also shown to reduce the adhesion and metastasis of prostate, lung, breast, and pancreas cancer cells by suppressing NF- $\kappa \mathrm{B}$, and protein kinase B transcription factors [114-116]. The combination of genistein with sorafenib was shown to improve the sensitivity of sorafenib-resistant hepatocellular carcinoma cell lines [111]. Several reports also indicate that genistein provides successful synergy when used in combination with tamoxifen in mammary tumors [117], with methotrexate in leukemia [118], and with cisplatin in ovarian cancer [119].

\section{$\operatorname{miR}-497$}

miR-497 mainly functions as a tumor suppressor gene reduced in many types of cancer. Its alteration by transcriptional factors and genomic and epigenetic modifications affects the proliferation, angiogenesis, and apoptosis of cancer cells [120]. miR-497 has been recognized to be a negative regulator of cisplatin resistance in ovarian cancer [121] and cervical cancer [122]. The enhanced expression of miR-497 sensitizes HeLa cells to cisplatin treatment. Upon further investigation, miR-497 was found to be a direct inhibitor of its downstream target TKT enzyme (Yang et al. [122]). Similarly, treatment of $\mathrm{HeLa}$ and SiHa cervical cancer cells with miR-497 significantly downregulated both mRNA and protein expression levels of TKT. Mechanistically, these findings were explained by miR-497 inhibitory effect on TKT, causing a significant reduction in GSH levels and hence a compromised detoxifying ability. Notably, miR-497 has been used in conjunction with many chemotherapeutic drugs to enhance their effect on cancer cells, namely, in combination with 5-fluorouracil (5-FU) in colorectal cancer [123] and re-sensitized pancreatic cancer to gemcitabine and erlotinib [124].

\section{TALDO Inhibitors}

TALDO catalyzes the second reaction of the nonoxidative PPP driving the conversion of G3P and sedoheptulose-7-phosphate into F6P and erythrose-4-phosphate.
TALDO is upregulated and implicated in cancer progression [125]. In Caenorhabditis elegans, TALDO inhibition reduced mitochondrial respiration, activated mitochondrial unfolded protein response, and induced longevity responses [126]. Similarly, mammalian mitochondrial function is also impacted when deficient for TALDO [127]. Patients with deficient TALDO showed reduced mitochondrial membrane potential, augmented mitochondrial mass, and elevated $\mathrm{H}_{2} \mathrm{O}_{2}$ levels in their isolated lymphoblasts [128]. Moreover, TALDO-deficient mice were infertile due to impaired mitochondria in spermatozoa [127].

\section{Fructose 1,6-Bisphosphate}

Fructose 1,6-bisphosphate is an intermediate in the glycolytic pathway that has been shown to inhibit the activity of TALDO. In E. coli, fructose 1,6-bisphosphate has been demonstrated to have 2 binding sites on TALDO; competing with F6P but not with erythrose-4-phosphate [129]. Moreover, this key enzyme of the nonoxidative PPP can be inhibited by different phosphorus compounds. For instance, arabinose 5-phosphate, an aldopentose version of the substrate F6P that lacks the 1-carbon and 1-hydroxyl atoms [130], has been implicated in suppressing cancer progression in murine colon cancer [131]. In addition, the monosaccharide D-tagatose 6-phosphate which has several applications as antidiabetic [132], and antioxidant medication [133] has been shown to irreversibly inhibit TALDO [134]. These compounds have strong similar stereochemistry with F6P but form Schiff bases with the active site lysine on TALDO and proceed to inhibit its activity [129].

\section{Conclusion}

Cancer cell metabolism is driven by extensive cellular proliferation. The PPP is upregulated in many tumors whereby its major enzymes may represent targets for novel anticancer regimen. Intracellular NADPH is mainly produced by the PPP and is required for redox homeostasis maintenance, which is needed to combat oxidative stress. In this review, we explored different regulators and exogenous inhibitors of the PPP in various cancer cells. The PPP enzymes are major players in several human diseases, with G6PD deficiency being the mostly reported metabolic disorder. However, G6PD, the rate-limiting PPP enzyme, plays a pro-survival role in cancer affecting cell proliferation and cell death. In diabetes, the nuclear factor E2-related factor 2, an oxidative stress regulated 
transcription factor, increased the expression of major PPP enzymes to promote cellular metabolism and contributed to cancer development [135]. PPP inhibitors, alone or in combination with conventional therapies, offer new treatment strategies especially in chemotherapyresistant patients. For instance, cisplatin resistance has been linked to the PPP oxidative phase upregulation. Therefore, the inhibition of the PPP in combination with cisplatin-sensitized tumor cells to chemotherapy [45]. Furthermore, the combination treatment with PPP inhibitors and 5-FU-sensitized 5-FU-resistant colorectal cancer cells to chemotherapy (unpublished data). p53, the most frequently inactivated gene in cancers, blocks PPP through the inhibition of G6PD dimerization and activation [27]. Restoring p53 function is a promising strategy in cancer therapy that aims at inhibiting PPP and therefore cancer cell growth [136]. In summary, although many anticancer molecules targeting the PPP are being currently investigated, more research is required to identify novel oxidative and nonoxidative PPP inhibitors from natural or synthetic sources that can offer better efficiency and specificity and less toxicity. The surge of nu- merous studies demonstrating how cancer cells hijack the PPP to optimize their survival highlights the importance of integrating PPP inhibitors in cancer therapy.

\section{Conflict of Interest Statement}

The authors have no conflicts of interest to declare.

\section{Funding Sources}

This research was supported by the American University of Beirut Medical Practice Plan and the American University of Beirut University Research Board.

\section{Author Contributions}

N.G., C.E.-B., and N.D. contributed to conception and design. N.G., C.E.-B., K.A., R.E.-K., and N.D. contributed to writing original. N.G., C.E.-B., and N.D. contributed to draft preparation. N.G., C.E.-B., K.A., R.E.-K., J.U., and N.D. contributed to revision.

\section{References}

1 Phan LM, Yeung SC, Lee MH. Cancer metabolic reprogramming: importance, main features, and potentials for precise targeted anticancer therapies. Cancer Biol Med. 2014; 11(1):1-19.

2 Hanahan D, Weinberg RA. Hallmarks of cancer: the next generation. Cell. 2011;144(5): 646-74.

3 Hanahan D, Weinberg RA. The hallmarks of cancer. Cell. 2000;100(1):57-70.

4 Darwiche N. Epigenetic mechanisms and the hallmarks of cancer: an intimate affair. Am J Cancer Res. 2020;10(7):1954-78.

5 Faubert B, Solmonson A, DeBerardinis RJ. Metabolic reprogramming and cancer progression. Science. 2020;368(6487):eaaw5473.

6 Cairns RA, Harris IS, Mak TW. Regulation of cancer cell metabolism. Nat Rev Cancer. 2011;11(2):85-95.

7 Hay N. Reprogramming glucose metabolism in cancer: can it be exploited for cancer therapy? Nat Rev Cancer. 2016;16(10):635-49.

8 Yoo HC, Yu YC, Sung Y, Han JM. Glutamine reliance in cell metabolism. Exp Mol Med. 2020;52(9):1496-516.

9 Martinez-Outschoorn UE, Peiris-Pagés M, Pestell RG, Sotgia F, Lisanti MP. Cancer metabolism: a therapeutic perspective. Nat Rev Clin Oncol. 2017;14(1):11-31.

10 Liberti MV, Locasale JW. Metabolism: a new layer of glycolysis. Nat Chem Biol. 2016;12(8): $577-8$.
11 Jin L, Zhou Y. Crucial role of the pentose phosphate pathway in malignant tumors. Oncol Lett. 2019;17(5):4213-21.

12 Pavlova NN, Thompson CB. The emerging hallmarks of cancer metabolism. Cell Metab. 2016;23(1):27-47.

13 Warburg O, Wind F, Negelein E. The metabolism of tumors in the body. J Gen Physiol. 1927;8(6):519-30.

14 Bader DA, McGuire SE. Tumour metabolism and its unique properties in prostate adenocarcinoma. Nat Rev Urol. 2020;17(4):214-31.

15 Schiliro C, Firestein BL. Mechanisms of metabolic reprogramming in cancer cells supporting enhanced growth and proliferation. Cells. 2021;10(5): 1056

16 Cho ES, Cha YH, Kim HS, Kim NH, Yook JI. The pentose phosphate pathway as a potential target for cancer therapy. Biomol Ther. 2018; 26(1):29-38.

17 Patra KC, Hay N. The pentose phosphate pathway and cancer. Trends Biochem Sci. 2014;39(8):347-54.

18 Dore MP, Davoli A, Longo N, Marras G, Pes GM. Glucose-6-phosphate dehydrogenase deficiency and risk of colorectal cancer in Northern Sardinia: a retrospective observational study. Medicine. 2016;95(44):e5254.

19 Kowalik MA, Columbano A, Perra A. Emerging role of the pentose phosphate pathway in hepatocellular carcinoma. Front Oncol. 2017; $7: 87$.
20 Lu SC. Glutathione synthesis. Biochim Biophys Acta. 2013;1830(5):3143-53.

21 Chen M, Shen M, Li Y, Liu C, Zhou K, Hu W, et al. GC-MS-based metabolomic analysis of human papillary thyroid carcinoma tissue. Int J Mol Med. 2015;36(6):1607-14.

22 Cohen HJ, Elizalde A, Miller SP. Cytologic studies of glucose-6-phosphate dehydrogenase in malignancy. Cancer. 1968;21(6): 1055-60.

23 Langbein S, Frederiks WM, zur Hausen A, Popa J, Lehmann J, Weiss C, et al. Metastasis is promoted by a bioenergetic switch: new targets for progressive renal cell cancer. Int J Cancer. 2008;122(11):2422-8.

24 Zampella EJ, Bradley EL Jr, Pretlow TG 2nd. Glucose-6-phosphate dehydrogenase: a possible clinical indicator for prostatic carcinoma. Cancer. 1982;49(2):384-7.

25 Stanton RC, Seifter JL, Boxer DC, Zimmerman E, Cantley LC. Rapid release of bound glucose-6-phosphate dehydrogenase by growth factors. Correlation with increased enzymatic activity. J Biol Chem. 1991;266(19): 12442-8.

26 Tian WN, Pignatare JN, Stanton RC. Signal transduction proteins that associate with the platelet-derived growth factor (PDGF) receptor mediate the PDGF-induced release of glucose-6-phosphate dehydrogenase from permeabilized cells. J Biol Chem. 1994;269(20): 14798-805. 
27 Jiang P, Du W, Wang X, Mancuso A, Gao X, $\mathrm{Wu} \mathrm{M}$, et al. p53 regulates biosynthesis through direct inactivation of glucose-6-phosphate dehydrogenase. Nat Cell Biol. 2011; 13(3):310-6.

28 Sukhatme VP, Chan B. Glycolytic cancer cells lacking 6-phosphogluconate dehydrogenase metabolize glucose to induce senescence. FEBS Lett. 2012;586(16):2389-95.

29 Ying H, Kimmelman AC, Lyssiotis CA, Hua S, Chu GC, Fletcher-Sananikone E, et al. Oncogenic Kras maintains pancreatic tumors through regulation of anabolic glucose metabolism. Cell. 2012;149(3):656-70.

30 Langbein S, Zerilli M, Zur Hausen A, Staiger W, Rensch-Boschert K, Lukan N, et al. Expression of transketolase TKTL1 predicts colon and urothelial cancer patient survival: Warburg effect reinterpreted. Br J Cancer. 2006; 94(4):578-85.

31 Basta PV, Bensen JT, Tse CK, Perou CM, Sullivan $\mathrm{PF}$, Olshan AF. Genetic variation in Transaldolase 1 and risk of squamous cell carcinoma of the head and neck. Cancer Detect Prev. 2008;32(3):200-8.

32 Wang C, Guo K, Gao D, Kang X, Jiang K, Li Y, et al. Identification of transaldolase as a novel serum biomarker for hepatocellular carcinoma metastasis using xenografted mouse model and clinic samples. Cancer Lett. 2011; 313(2):154-66.

33 Jiang P, Du W, Wu M. Regulation of the pentose phosphate pathway in cancer. Protein Cell. 2014;5(8):592-602.

34 Chen L, Zhang Z, Hoshino A, Zheng HD, Morley M, Arany Z, et al. NADPH production by the oxidative pentose-phosphate pathway supports folate metabolism. Nat Metab. 2019; 1(3):404-15.

35 Lowman XH, McDonnell MA, Kosloske A, Odumade OA, Jenness C, Karim CB, et al. The proapoptotic function of noxa in human leukemia cells is regulated by the kinase Cdk5 and by glucose. Mol Cell. 2010;40(5):823-33.

36 Ge T, Yang J, Zhou S, Wang Y, Li Y, Tong X. The role of the pentose phosphate pathway in diabetes and cancer. Front Endocrinol. 2020; 11:365.

37 Bensaad K, Tsuruta A, Selak MA, Vidal MN, Nakano K, Bartrons R, et al. TIGAR, a p53inducible regulator of glycolysis and apoptosis. Cell. 2006;126(1):107-20.

38 Zhang X, Zhang X, Li Y, Shao Y, Xiao J, Zhu $\mathrm{G}$, et al. PAK4 regulates G6PD activity by $\mathrm{p} 53$ degradation involving colon cancer cell growth. Cell Death Dis. 2017;8(5):e2820.

39 Düvel K, Yecies JL, Menon S, Raman P, Lipovsky AI, Souza AL, et al. Activation of a metabolic gene regulatory network downstream of mTOR complex 1. Mol Cell. 2010; 39(2):171-83.

40 Shibuya N, Inoue K, Tanaka G, Akimoto K, Kubota K. Augmented pentose phosphate pathway plays critical roles in colorectal carcinomas. Oncology. 2015;88(5):309-19.
41 Chen H, Wu D, Bao L, Yin T, Lei D, Yu J, et al. 6PGD inhibition sensitizes hepatocellular carcinoma to chemotherapy via AMPK activation and metabolic reprogramming. Biomed Pharmacother. 2019;111:1353-8.

42 Shimizu T, Inoue K, Hachiya $\mathrm{H}$, Shibuya $\mathrm{N}$, Shimoda M, Kubota K. Frequent alteration of the protein synthesis of enzymes for glucose metabolism in hepatocellular carcinomas. J Gastroenterol. 2014;49(9):1324-32.

43 Vaghari-Tabari M, Ferns GA, Qujeq D, Andevari AN, Sabahi Z, Moein S. Signaling, metabolism, and cancer: an important relationship for therapeutic intervention. J Cell Physiol. 2021;236(8):5512-32.

44 Mele L, Paino F, Papaccio F, Regad T, Boocock $\mathrm{D}$, Stiuso $\mathrm{P}$, et al. A new inhibitor of glucose6-phosphate dehydrogenase blocks pentose phosphate pathway and suppresses malignant proliferation and metastasis in vivo. Cell Death Dis. 2018;9(5):572.

45 Giacomini I, Ragazzi E, Pasut G, Montopoli $M$. The pentose phosphate pathway and its involvement in cisplatin resistance. Int J Mol Sci. 2020;21(3):937.

46 Yang HC, Stern A, Chiu DT. G6PD: a hub for metabolic reprogramming and redox signaling in cancer. Biomed J. 2021;44(3):285-92.

47 Zhang J, Zhou L, Zhang P, Liu T, Yang G, Lin $\mathrm{R}$, et al. Extraction of polydatin and resveratrol from polygonum cuspidatum root: kinetics and modeling. Food Bioprod Processing. 2015;94:518-24.

48 Zhao J, Li H, Wang Z, Tong C. Effect of polydatin on ultrastructure of cardiac myocytes in rats with adriamycin-induced myocardial damage [J]. Acta Academiae Medicinae CPAF. 2010;8:15.

49 Lanzilli G, Cottarelli A, Nicotera G, Guida S, Ravagnan G, Fuggetta MP. Anti-inflammatory effect of resveratrol and polydatin by in vitro IL-17 modulation. Inflammation. 2012; 35(1):240-8.

50 Yuan M, Li J, Lv J, Mo X, Yang C, Chen X, et al. Polydatin (PD) inhibits IgE-mediated passive cutaneous anaphylaxis in mice by stabilizing mast cells through modulating $\mathrm{Ca} 2+$ mobilization. Toxicol Appl Pharmacol. 2012; 264(3):462-9.

51 Mikulski D, Molski M. Quantitative structureantioxidant activity relationship of trans-resveratrol oligomers, trans- $4,4^{\prime}$ dihydroxystilbene dimer, trans-resveratrol3-O-glucuronide, glucosides: trans-piceid, cis-piceid, trans-astringin and transresveratrol-4'-O-beta-D-glucopyranoside. Eur J Med Chem. 2010;45(6):2366-80.

52 Mele L, la Noce M, Paino F, Regad T, Wagner S, Liccardo D, et al. Glucose-6-phosphate dehydrogenase blockade potentiates tyrosine kinase inhibitor effect on breast cancer cells through autophagy perturbation. J Exp Clin Cancer Res. 2019;38(1):160.
53 De Maria S, Scognamiglio I, Lombardi A, Amodio N, Caraglia M, Cartenì M, et al. Polydatin, a natural precursor of resveratrol, induces cell cycle arrest and differentiation of human colorectal Caco-2 cell. J Transl Med. 2013;11:264.

54 Indraccolo U, Indraccolo SR, Mignini F. Micronized palmitoylethanolamide/trans-polydatin treatment of endometriosis-related pain: a meta-analysis. Ann Ist Super Sanita. 2017;53(2):125-34.

55 Klinge CM, Clark BJ, Prough RA. Dehydroepiandrosterone research: past, current, and future. Vitam Horm. 2018;108:1-28.

56 Di Monaco M, Pizzini A, Gatto V, Leonardi L, Gallo M, Brignardello E, et al. Role of glucose6-phosphate dehydrogenase inhibition in the antiproliferative effects of dehydroepiandrosterone on human breast cancer cells. Br J Cancer. 1997;75(4):589-92.

57 Tsutsui EA, Marks PA, Reich P. Effect of dehydroepiandrosterone on glucose 6-phosphate dehydrogenase activity and reduced triphosphopyridine nucleotide formation in adrenal tissue. J Biol Chem. 1962;237:3009-13.

58 Jo H, Park JS, Kim EM, Jung MY, Lee SH, Seong SC, et al. The in vitro effects of dehydroepiandrosterone on human osteoarthritic chondrocytes. Osteoarthritis Cartilage. 2003; 11(8):585-94.

59 Savineau JP, Marthan R, Dumas de la Roque E. Role of DHEA in cardiovascular diseases. Biochem Pharmacol. 2013;85(6):718-26.

60 Zumoff B, Levin J, Rosenfeld RS, Markham M, Strain GW, Fukushima DK. Abnormal 24-hr mean plasma concentrations of dehydroisoandrosterone and dehydroisoandrosterone sulfate in women with primary operable breast cancer. Cancer Res. 1981;41(9 Pt 1):3360-3.

61 Gordon G, Mackow MC, Levy HR. On the mechanism of interaction of steroids with human glucose 6-phosphate dehydrogenase. Arch Biochem Biophys. 1995;318(1):25-9.

62 Liu CL, Hsu YC, Lee JJ, Chen MJ, Lin CH, Huang SY, et al. Targeting the pentose phosphate pathway increases reactive oxygen species and induces apoptosis in thyroid cancer cells. Mol Cell Endocrinol. 2020;499:110595.

63 Herken H. Biosynthesis and action of dinucleotides containing 6-aminonicotinamide on membrane transport processes. Arzneimittelforschung. 1968;18(10):1235-45.

64 Carmona A, Freedland RA. Effect of 6-aminonicotinamide on pentose cycle activity in isolated hepatocytes. Int J Biochem. 1990;22(6): 595-9.

65 Dietrich LS, Friedland IM, Kaplan LA. Pyridine nucleotide metabolism: mechanism of action of the niacin antagonist, 6-aminonicotinamide. J Biol Chem. 1958;233(4):964-8.

66 Budihardjo II, Walker DL, Svingen PA, Buckwalter CA, Desnoyers S, Eckdahl S, et al. 6-Aminonicotinamide sensitizes human tumor cell lines to cisplatin. Clin Cancer Res. 1998;4(1):117-30. 
67 Varnes ME. Inhibition of pentose cycle of A549 cells by 6 -aminonicotinamide: consequences for aerobic and hypoxic radiation response and for radiosensitizer action. NCI Monogr. 1988(6):199-203

68 Koutcher JA, Alfieri AA, Stolfi RL, Devitt ML, Colofiore JR, Nord LD, et al. Potentiation of a three drug chemotherapy regimen by radiation. Cancer Res. 1993;53(15):3518-23.

69 Belfi CA, Chatterjee S, Gosky DM, Berger SJ, Berger NA. Increased sensitivity of human colon cancer cells to DNA cross-linking agents after GRP78 up-regulation. Biochem Biophys Res Commun. 1999;257(2):361-8.

70 Stolfi RL, Colofiore JR, Nord LD, Koutcher JA, Martin DS. Biochemical modulation of tumor cell energy: regression of advanced spontaneous murine breast tumors with a 5 -fluorouracil-containing drug combination. Cancer Res. 1992;52(15):4074-81.

71 Dietrich LS, Muniz O, Farinas B, Franklin L. 6-aminonicotinamide-14C utilization by the 755 tumor and host liver tissue. Cancer Res. 1968;28(8):1652-4.

72 Polimeni M, Voena C, Kopecka J, Riganti C, Pescarmona G, Bosia A, et al. Modulation of doxorubicin resistance by the glucose-6-phosphate dehydrogenase activity. Biochem J. 2011; 439(1):141-9.

73 Ren F, Yang X, Hu ZW, Wong VKW, Xu HY, Ren JH, et al. Niacin analogue, 6-Aminonicotinamide, a novel inhibitor of hepatitis $B$ virus replication and HBsAg production. EBioMedicine. 2019;49:232-46.

74 Penkowa M, Quintana A, Carrasco J, Giralt M, Molinero A, Hidalgo J. Metallothionein prevents neurodegeneration and central nervous system cell death after treatment with gliotoxin 6-aminonicotinamide. J Neurosci Res. 2004 77(1):35-53.

75 Torretta S, Scagliola A, Ricci L, Mainini F, Di Marco S, Cuccovillo I, et al. D-mannose suppresses macrophage IL-1 $\beta$ production. Nat Commun. 2020;11(1):6343.

76 Sharma V, Ichikawa M, Freeze HH. Mannose metabolism: more than meets the eye. Biochem Biophys Res Commun. 2014;453(2):220-8.

77 Liu F, Xu X, Li C, Li C, Li Y, Yin S, et al. Mannose synergizes with chemoradiotherapy to cure cancer via metabolically targeting HIF- 1 in a novel triple-negative glioblastoma mouse model. Clin Transl Med. 2020;10(7):e226.

78 Gonzalez PS, O’Prey J, Cardaci S, Barthet VJA, Sakamaki JI, Beaumatin F, et al. Mannose impairs tumour growth and enhances chemotherapy. Nature. 2018;563(7733):719-23.

79 Wang Y, Xie S, He B. Mannose shows antitumour properties against lung cancer via inhibiting proliferation, promoting cisplatin-mediated apoptosis and reducing metastasis. $\mathrm{Mol}$ Med Rep. 2020;22(4):2957-65.

80 Dalle Vedove E, Costabile G, Merkel OM Mannose and mannose-6-phosphate receptortargeted drug delivery systems and their application in cancer therapy. Adv Healthc Mater. 2018;7(14):e1701398.
$81 \mathrm{Hu}$ J, Wei P, Seeberger PH, Yin J. Mannosefunctionalized nanoscaffolds for targeted delivery in biomedical applications. Chem Asian J. 2018;13(22):3448-59.

82 Theologides A. Anorexia-producing intermediary metabolites. Am J Clin Nutr. 1976;29(5): 552-8

83 Deane EE, Woo NY. Expression studies on glucose-6-phosphate dehydrogenase in sea bream: effects of growth hormone, somatostatin, salinity and temperature. J Exp Zool A Comp Exp Biol. 2005;303(8):676-88.

84 Boros LG, Brandes JL, Yusuf FI, Cascante M, Williams RD, Schirmer WJ. Inhibition of the oxidative and nonoxidative pentose phosphate pathways by somatostatin: a possible mechanism of antitumor action. Med Hypotheses. 1998;50(6):501-6.

85 Liu C, Zhang S, Wang Q, Zhang X. Tumor suppressor miR-1 inhibits tumor growth and metastasis by simultaneously targeting multiple genes. Oncotarget. 2017;8(26):42043-60.

$86 \mathrm{Hu} \mathrm{T}$, Chang YF, Xiao Z, Mao R, Tong J, Chen $B$, et al. miR-1 inhibits progression of high-risk papillomavirus-associated human cervical cancer by targeting G6PD. Oncotarget. 2016;7(52): 86103-16.

87 Wang L, Yuan Y, Li J, Ren H, Cai Q, Chen X, et al. MicroRNA-1 aggravates cardiac oxidative stress by post-transcriptional modification of the antioxidant network. Cell Stress Chaperones. 2015;20(3):411-20.

88 Barajas JM, Reyes R, Guerrero MJ, Jacob ST, Motiwala T, Ghoshal K. The role of miR-122 in the dysregulation of glucose-6-phosphate dehydrogenase (G6PD) expression in hepatocellular cancer. Sci Rep. 2018;8(1):9105.

89 Bravard A, Luccioni C, Muleris M, Lefrancois D, Dutrillaux B. Relationships between UMPK and PGD activities and deletions of chromosome 1p in colorectal cancers. Cancer Genet Cytogenet. 1991;56(1):45-56.

90 Basu J, Duttagupta C, Vermund SH, Ahn C, Palan PR, Romney SL. Alterations in erythrocyte glutathione metabolism associated with cervical dysplasias and carcinoma in situ. Cancer Invest. 1993;11(6):652-9.

91 Giusti L, Iacconi P, Ciregia F, Giannaccini G, Donatini GL, Basolo F, et al. Fine-needle aspiration of thyroid nodules: proteomic analysis to identify cancer biomarkers. J Proteome Res. 2008;7(9):4079-88.

92 Lin R, Elf S, Shan C, Kang HB, Ji Q, Zhou L, et al. 6-Phosphogluconate dehydrogenase links oxidative PPP, lipogenesis and tumour growth by inhibiting LKB1-AMPK signalling. Nat Cell Biol. 2015;17(11):1484-96.

93 Guo H, Xiang Z, Zhang Y, Sun D. Inhibiting 6-phosphogluconate dehydrogenase enhances chemotherapy efficacy in cervical cancer via AMPK-independent inhibition of RhoA and Rac1. Clin Transl Oncol. 2019;21(4):404-11.

94 Pan X, Wang H, Tong D, Wang C, Sun L, Zhao $\mathrm{C}$, et al. Physcion induces apoptosis in hepatocellular carcinoma by modulating miR-370. Am J Cancer Res. 2016;6(12):2919-31.
95 Hitosugi T, Zhou L, Elf S, Fan J, Kang HB, Seo $\mathrm{JH}$, et al. Phosphoglycerate mutase 1 coordinates glycolysis and biosynthesis to promote tumor growth. Cancer Cell. 2012;22(5):585-600.

96 Zheng W, Feng Q, Liu J, Guo Y, Gao L, Li R et al. Inhibition of 6-phosphogluconate dehydrogenase reverses cisplatin resistance in ovarian and lung cancer. Front Pharmacol. 2017;8:421.

97 Xu IM, Lai RK, Lin SH, Tse AP, Chiu DK, Koh HY, et al. Transketolase counteracts oxidative stress to drive cancer development. Proc Natl Acad Sci U S A. 2016;113(6):E725-34.

98 Comín-Anduix B, Boren J, Martinez S, Moro C, Centelles JJ, Trebukhina R, et al. The effect of thiamine supplementation on tumour proliferation. A metabolic control analysis study. Eur J Biochem. 2001;268(15):4177-82.

99 Brin M. Effects of thiamine deficiency and of oxythiamine on rat tissue transketolase. J Nutr. 1962;78(2):179-83.

100 Wang J, Zhang X, Ma D, Lee WP, Xiao J, Zhao $\mathrm{Y}$, et al. Inhibition of transketolase by oxythiamine altered dynamics of protein signals in pancreatic cancer cells. Exp Hematol Oncol. 2013;2:18.

101 Mariadasse R, Biswal J, Jayaprakash P, Rao GR, Choubey SK, Rajendran S, et al. Mechanical insights of oxythiamine compound as potent inhibitor for human transketolase-like protein 1 (TKTL1 protein). J Recept Signal Transduct Res. 2016;36(3):233-42.

102 Siemieniuk M, Sosnowska K, Czerniecki J, Czyzewska U, Winnicka K, Tylicki A. Oxythiamine improves antifungal activity of ketoconazole evaluated in canine Malassezia pachydermatis strains. Vet Dermatol. 2018; 29(6):476-e160.

103 Raïs B, Comin B, Puigjaner J, Brandes JL, Creppy E, Saboureau D, et al. Oxythiamine and dehydroepiandrosterone induce a G1 phase cycle arrest in Ehrlich's tumor cells through inhibition of the pentose cycle. FEBS Lett. 1999;456(1):113-8.

104 Zhang H, Cao R, Lee WN, Deng C, Zhao Y, Lappe J, et al. Inhibition of protein phosphorylation in MIA pancreatic cancer cells: confluence of metabolic and signaling pathways. J Proteome Res. 2010;9(2):980-9.

105 Yang CM, Liu YZ, Liao JW, Hu ML. The in vitro and in vivo anti-metastatic efficacy of oxythiamine and the possible mechanisms of action. Clin Exp Metastasis. 2010;27(5):341-9.

106 Wang CY, Shui HA, Chang TC. Dual effects for lovastatin in anaplastic thyroid cancer: the pivotal effect of transketolase (TKT) on lovastatin and tumor proliferation. J Investig Med. 2018;66(5):1-9.

107 Su SJ, Yeh TM, Lei HY, Chow NH. The potential of soybean foods as a chemoprevention approach for human urinary tract cancer. Clin Cancer Res. 2000;6(1):230-6.

108 Taylor CK, Levy RM, Elliott JC, Burnett BP. The effect of genistein aglycone on cancer and cancer risk: a review of in vitro, preclinical, and clinical studies. Nutr Rev. 2009;67(7): $398-415$. 
109 Tuli HS, Tuorkey MJ, Thakral F, Sak K, Kumar $\mathrm{M}$, Sharma AK, et al. Molecular mechanisms of action of genistein in cancer: recent advances. Front Pharmacol. 2019;10:1336.

110 Uckun FM, Messinger Y, Chen CL, O’Neill K, Myers DE, Goldman F, et al. Treatment of therapy-refractory B-lineage acute lymphoblastic leukemia with an apoptosis-inducing CD19directed tyrosine kinase inhibitor. Clin Cancer Res. 1999;5(12):3906-13.

111 Li S, Li J, Dai W, Zhang Q, Feng J, Wu L, et al. Genistein suppresses aerobic glycolysis and induces hepatocellular carcinoma cell death. Br J Cancer. 2017;117(10):1518-28.

112 Boros LG, Bassilian S, Lim S, Lee WN. Genistein inhibits nonoxidative ribose synthesis in MIA pancreatic adenocarcinoma cells: a new mechanism of controlling tumor growth. Pancreas. 2001;22(1):1-7.

113 Regenbrecht CR, Jung M, Lehrach H, Adjaye J. The molecular basis of genistein-induced mitotic arrest and exit of self-renewal in embryonal carcinoma and primary cancer cell lines. BMC Med Genomics. 2008;1(1):49.

114 Gong L, Li Y, Nedeljkovic-Kurepa A, Sarkar FH. Inactivation of NF-kappaB by genistein is mediated via Akt signaling pathway in breast cancer cells. Oncogene. 2003;22(30):4702-9.

115 Li Y, Ellis KL, Ali S, El-Rayes BF, NedelikovicKurepa A, Kucuk O, et al. Apoptosis-inducing effect of chemotherapeutic agents is potentiated by soy isoflavone genistein, a natural inhibitor of NF-kappaB in BxPC-3 pancreatic cancer cell line. Pancreas. 2004;28(4):e90-5.

116 Li Y, Ahmed F, Ali S, Philip PA, Kucuk O, Sarkar FH. Inactivation of nuclear factor kap$\mathrm{paB}$ by soy isoflavone genistein contributes to increased apoptosis induced by chemotherapeutic agents in human cancer cells. Cancer Res. 2005;65(15):6934-42.

117 Zhang X, Cook KL, Warri A, Cruz IM, Rosim $\mathrm{M}$, Riskin J, et al. Lifetime genistein intake increases the response of mammary tumors to tamoxifen in rats. Clin Cancer Res. 2017;23(3): 814-24.

118 Liu T, Singh R, Rios Z, Bhushan A, Li M, Sheridan PP, et al. Tyrosine phosphorylation of HSC70 and its interaction with RFC mediates methotrexate resistance in murine L1210 leukemia cells. Cancer Lett. 2015;357(1):231-41.

119 Arzuman L, Beale P, Proschogo N, Yu JQ, Huq F. Combination of genistein and cisplatin with two designed monofunctional platinum agents in human ovarian tumour models. Anticancer Res. 2015;35(11):6027-39.

120 Yang G, Xiong G, Cao Z, Zheng S, You L, Zhang $\mathrm{T}$, et al. miR-497 expression, function and clinical application in cancer. Oncotarget. 2016; 7(34):55900-11.

$121 \mathrm{Xu}$ S, Fu GB, Tao Z, OuYang J, Kong F, Jiang $\mathrm{BH}$, et al. MiR-497 decreases cisplatin resistance in ovarian cancer cells by targeting mTOR/ P70S6K1. Oncotarget. 2015;6(28):26457-71.

122 Yang H, Wu XL, Wu KH, Zhang R, Ju LL, Ji Y, et al. MicroRNA-497 regulates cisplatin chemosensitivity of cervical cancer by targeting transketolase. Am J Cancer Res. 2016;6(11):2690-9.
123 Wang L, Jiang CF, Li DM, Ge X, Shi ZM, Li CY, et al. MicroRNA-497 inhibits tumor growth and increases chemosensitivity to 5-fluorouracil treatment by targeting KSR1. Oncotarget. 2016;7(3):2660-71.

124 Xu J, Wang T, Cao Z, Huang H, Li J, Liu W, et al. MiR-497 downregulation contributes to the malignancy of pancreatic cancer and associates with a poor prognosis. Oncotarget. 2014;5(16): 6983-93.

125 Lachaise F, Martin G, Drougard C, Perl A, Vuillaume M, Wegnez M, et al. Relationship between posttranslational modification of transaldolase and catalase deficiency in UV-sensitive repair-deficient xeroderma pigmentosum fibroblasts and SV40-transformed human cells. Free Radic Biol Med. 2001;30(12):1365-73.

126 Bennett CF, Kwon JJ, Chen C, Russell J, Acosta $\mathrm{K}$, Burnaevskiy N, et al. Transaldolase inhibition impairs mitochondrial respiration and induces a starvation-like longevity response in Caenorhabditis elegans. PLoS Genet. 2017; 13(3):e1006695.

127 Perl A, Qian Y, Chohan KR, Shirley CR, Amidon $\mathrm{W}$, Banerjee $\mathrm{S}$, et al. Transaldolase is essential for maintenance of the mitochondrial transmembrane potential and fertility of spermatozoa. Proc Natl Acad Sci U S A. 2006;103(40): 14813-8.

128 Qian Y, Banerjee S, Grossman CE, Amidon W, Nagy G, Barcza M, et al. Transaldolase deficiency influences the pentose phosphate pathway, mitochondrial homoeostasis and apoptosis signal processing. Biochem J. 2008;415(1):123-34.

129 Ogawa T, Murakami K, Yoshino M. Inhibition by fructose 1,6-bisphosphate of transaldolase from Escherichia coli. FEMS Microbiol Lett. 2016;363(17).

130 Light SH, Anderson WF. Arabinose 5-phosphate covalently inhibits transaldolase. J Struct Funct Genomics. 2014;15(1):41-4.

131 Hong H, Lim D, Kim GJ, Park SH, Sik Kim H, Hong Y, et al. Targeted deletion of the ara operon of Salmonella typhimurium enhances Larabinose accumulation and drives PBAD-promoted expression of anti-cancer toxins and imaging agents. Cell Cycle. 2014;13(19):3112-20.

132 Lu Y, Levin GV, Donner TW. Tagatose, a new antidiabetic and obesity control drug. Diabetes Obes Metab. 2008;10(2):109-34.

133 Paterna JC, Boess F, Stäubli A, Boelsterli UA Antioxidant and cytoprotective properties of Dtagatose in cultured murine hepatocytes. Toxicol Appl Pharmacol. 1998;148(1):117-25.

134 Stellmacher L, Sandalova T, Schneider S, Schneider G, Sprenger GA, Samland AK. Novel mode of inhibition by D-tagatose 6-phosphate through a Heyns rearrangement in the active site of transaldolase B variants. Acta Crystallogr D Struct Biol. 2016;72(Pt 4):467-76.

135 Mitsuishi Y, Taguchi K, Kawatani Y, Shibata T, Nukiwa T, Aburatani $\mathrm{H}$, et al. Nrf2 redirects glucose and glutamine into anabolic pathways in metabolic reprogramming. Cancer Cell. 2012;22(1):66-79.
136 Lozano G. Restoring p53 in cancer: the promises and the challenges. J Mol Cell Biol. 2019; 11(7):615-9.

137 Zhu Y, Qiu L, Jiang F, Găman MA, Abudoraehem OS, Okunade KS, et al. The effect of dehydroepiandrosterone (DHEA) supplementation on estradiol levels in women: a dose-response and meta-analysis of randomized clinical trials. Steroids. 2021;173:108889.

138 Herter FP, Weissman SG, Thompson HG Jr, Hyman G, Martin DS. Clinical experience with 6-aminonicotinamide. Cancer Res. 1961;21: 31-7.

139 Gomes-Porras M, Cárdenas-Salas J, ÁlvarezEscolá C. Somatostatin analogs in clinical practice: a review. Int J Mol Sci. 2020;21(5):1682.

140 Han C, Yu Z, Duan Z, Kan Q. Role of microRNA-1 in human cancer and its therapeutic potentials. Biomed Res Int. 2014;2014:428371.

$141 \mathrm{He}$ C, Yang J, Ding J, Li S, Wu H, Xiong Y, et al. Downregulation of glucose-6-phosphate dehydrogenase by microRNA-1 inhibits the growth of pituitary tumor cells. Oncol Rep. 2018;40(6): 3533-42.

142 Kura B, Kalocayova B, Devaux Y, Bartekova M Potential clinical implications of miR-1 and miR-21 in heart disease and cardioprotection. Int J Mol Sci. 2020;21(3):700.

143 Hanna J, Hossain GS, Kocerha J. The potential for microRNA therapeutics and clinical research. Front Genet. 2019;10(478):478.

144 Pang MJ, Yang Z, Zhang XL, Liu ZF, Fan J, Zhang HY. Physcion, a naturally occurring anthraquinone derivative, induces apoptosis and autophagy in human nasopharyngeal carcinoma. Acta Pharmacol Sin. 2016;37(12):1623-40.

145 Sang Q, Liu X, Sun D. Role of miR-613 as a tumor suppressor in glioma cells by targeting SOX9. Onco Targets Ther. 2018;11:2429-38.

146 Zhang F, Masania J, Anwar A, Xue M, Zehnder $\mathrm{D}$, Kanji $\mathrm{H}$, et al. The uremic toxin oxythiamine causes functional thiamine deficiency in endstage renal disease by inhibiting transketolase activity. Kidney Int. 2016;90(2):396-403.

147 Lazarevic B, Boezelijn G, Diep LM, Kvernrod K, Ogren O, Ramberg H, et al. Efficacy and safety of short-term genistein intervention in patients with localized prostate cancer prior to radical prostatectomy: a randomized, placebo-controlled, double-blind Phase 2 clinical trial. Nutr Cancer. 2011;63(6):889-98.

148 Huang X, Wang L, Liu W, Li F. MicroRNA497-5p inhibits proliferation and invasion of non-small cell lung cancer by regulating FGF2. Oncol Lett. 2019;17(3):3425-31.

149 To KKW, Fong W, Tong CWS, Wu M, Yan W, Cho WCS. Advances in the discovery of microRNA-based anticancer therapeutics: latest tools and developments. Expert Opin Drug Discov. 2020;15(1):63-83.

150 Markov AK, Brumley MA, Figueroa A, Skelton TN, Lehan PH. Hemodynamic effects of fructose 1,6-diphosphate in patients with normal and impaired left ventricular function. Am Heart J. 1997;133(5):541-9.
Inhibitors of the Pentose Phosphate Pathway in Cancer
Chemotherapy 2021;66:179-191 DOI: $10.1159 / 000519784$ 